\title{
6-烷氧基-2-丙硫基-8-氮杂嘌呤核苷的合成及抗血小板凝聚活性评价
}

\author{
邓聪迩李顺来刘祥伟 杜洪光* \\ (北京化工大学理学院 北京 100029)
}

\begin{abstract}
摘要 2-硫代巴比妥酸(1)经过颈基的烷基化、嘧啶环硝化以及羟基的氯代三步反应得到 5-硝基-2-丙硫基-4,6-二氯嘧啶 (4)；1- $O$-乙酰基- $2,3,5$-三- $O$-苯甲酰基- $\beta$ - $D$-呋喃核糖(5)经过叠氮取代及叠氮的还原反应得到 1-氨基-2,3,5-三- $O$-苯甲酰 基- $\beta$ - $D$-呋喃核糖(7); 化合物 7 与 4 经过亲核取代、硝基还原、重氮化及偶联反应得到 9-[(2', 3', $5^{\prime}$-三- $O$-苯甲酰基)- $\beta$ - $D$ 呋喃核糖基]-2-丙硫基-6-氯-8-氮杂嘌呤(10); 化合物 10 与醇进行亲核取代及脱保护反应后，得到 10 个未见报道的 6-烷 氧基-2-丙硫基-8-氮杂嘌呤核苷化合物(11). 化合物的结构经过 ${ }^{1} \mathrm{H} N \mathrm{NM},{ }^{13} \mathrm{C} N \mathrm{NR}$, IR 以及 HRMS 得到表征, 并进行了 抗血小板凝聚活性测试.
\end{abstract}

关键词 8-氮杂嘌呤核苷; 合成; 结构表征; 抗血小板凝聚

\section{Synthesis of 6-Alkoxyl-2-propylthio-8-azapurine Nucleosides and Their Antiplatelet Aggregation Activity Evaluation}

\author{
Deng, Conger Li, Shunlai Liu, Xiangwei Du, Hongguang* \\ (College of Science, Beijing University of Chemical Technology, Beijing 100029)
}

\begin{abstract}
Thiobarbituric acid (1) was converted to 4,6-dichloro-5-nitro-2-propylthiopyrimidine (4) via S-alkylation, nitration and chlorination. Followed by azide and reduction reaction of azido, $1-O$-acetyl-2,3,5-tri- $O$-benzoyl- $\beta$ - $D$-ribofuranose (5) was converted to 1 -amino-2,3,5-tri- $O$-benzoyl- $\beta$ - $D$-ribofuranose (7). 9-[(2',3',5'-Tri- $O$-benzoyl)- $\beta$ - $D$-ribofuranosyl]-6-chloro2-propylthio-8-azapurine (10) was obtained via nucleophilic substitution of 7 with 4 , reduction, diazotization and coupling reaction. Nucleophilic displacement of the chloride in $\mathbf{1 0}$ with various alcohols and deprotection afforded 6-alkoxyl-2-propylthio-8-azapurine nucleosides (11). Their structures were identified by ${ }^{1} \mathrm{H}$ NMR, ${ }^{13} \mathrm{C} N M R$, IR and HRMS techniques. Moreover, the antiplatelet aggregation activities of compounds $\mathbf{1 1}$ were measured.

Keywords 8-azapurine nucleosides; synthesis; characterization; antiplatelet aggregation
\end{abstract}

血小板的粘附聚集对于血栓的形成起着重要的作 用 ${ }^{[1]}$. 在临床医学上, 抗血小板药物使用最广, 占抗血 栓药物的一半以上. 抗血小板药物治疗血栓病的作用机 理是通过抑制血小板活化，避免血小板粘附聚集起到抗 血栓效应. 目前临床医学上主要应用的抗血小板药物 有：血栓素抑制剂阿司匹林、二磷酸腺苷受体拮抗剂氯 吡格雷与噻氯吡啶、磷酸二酯酶抑制剂双嘧达莫以及血. 小板膜糖蛋白 IIb/IIIa 受体拮抗剂阿昔单抗和埃替巴肽

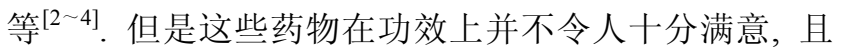
存在个体性差异以及副作用, 因此寻找高效稳定且副作 用小的新型抗血小板药物成为了人们研究的热点.

8-氮杂嘌呤核苷类化合物在临床医学上有着重要
的研究价值, 它在抗病毒 ${ }^{[5 \sim 7]}$ 、抗肿瘤 ${ }^{[8]}$ 和治疗血栓性疾 病方面 ${ }^{[9,10]}$ 都有着良好的医药活性. 近年来, 阿斯利康 公司通过对 8-氮杂嘌呤核苷核糖环的 5'-位进行官能团 修饰，以及对嘌呤环 2,6-位进行烷硫基或烷氨基取代， 合成了一系列 8-氮杂嘌呤核苷化合物, 并对这些化合物 进行了抗血小板聚集的笁选 ${ }^{[11,12]}$. AR-C78511 与 AZD6140 (Scheme 1)在篎选中都表现出了非常好的抗 血小板聚集活性. 尤其是 AZD6140, 作为一种新的 P2 $Y_{12}$ 拮抗剂, 对 ADP 引起的血小板聚集有明显的抑制 作用. 而 AR-C78511 则在具有出众的血小板聚集抑制 能力的同时, 还具有很好的反相激动性 ${ }^{[13]}$.

\footnotetext{
* E-mail: dhg@mail.buct.edu.cn

Received March 11, 2013; revised April 4, 2013; published online April 17, 2013.

Project supported by the National Natural Science Foundation of China (No. 21272022).

国家自然科学基金(No. 21272022)资助项目.
} 
<smiles>CCCCCCNc1nc(SCCC)nc2c1nnn2[C@@H]1O[C@@H](/C=C/C(=O)N[C@@H](CC(=O)O)C(=O)O)[C@H](O)[C@H]1O</smiles>

AR-C78511<smiles>CCCSc1nc(N[C@H]2CC2c2ccc(F)c(F)c2)c2nnn(C3CC(O)C(O)O3)c2n1</smiles>

AZD6140<smiles>[R]Oc1nc(SCCC)nc2c1nnn2C1OC2(O)COC1C2O</smiles>

Scheme 1

近年来, 本课题组对嘌呤衍生物 ${ }^{[14,15]}$ 和 8-氮杂嘌呤 衍生物的合成和抗血小板凝聚活性进行了研究. 到目前 为止，对 6-位为烷氧基的 8-氮杂嘌呤核苷的合成和生物 活性研究很少. 本文以 2-硫代巴比妥酸与 1- $O$-乙酰基$2,3,5$-三- $O$-苯甲酰基- $\beta$ - $D$-呋喃核糖为起始原料, 设计合 成了 10 种未见报道的 6-烷氧基-2-丙硫基-8-氮杂嘌呤核 苷化合物，通过 ${ }^{1} \mathrm{H}$ NMR, ${ }^{13} \mathrm{C}$ NMR, IR 和 HRMS 等对其 结构进行表征, 并且对这 10 种化合物进行了抗血小板
凝聚活性的测试. 合成路线如 Scheme 2 所示.

\section{1 实验部分}

\section{1 仪器与试剂}

XT-4A 型显微熔点测定仪, 上海精密科学仪器有限 公司(温度计未校正); Nicolet 5700 型傅里叶变换红外光 谱仪，美国 Thermo Electron Corporation; Bruker 400 $\mathrm{MHz}$ 核磁共振仪(TMS 为内标), 德国 Bruker 公司; Agilent TOF-6210 型高分辨质谱仪，美国 Agilent 公司; Chrono-Log Model 400VS 血小板聚集仪, 美国 ChronoLog 公司. 2-硫代巴比妥酸, 河南新乡金牛精细化工有 限公司；1- $O$-乙酰基-2,3,5-三- $O$-苯甲酰基- $\beta$ - $D$-呋喃核 糖，河南新乡一梅化工有限公司; 碘丙烷、无水四氯化 锡、三氯氧磷、 $N, N$-二异丙基乙胺等为分析纯.

\section{2 实验方法}

\subsubsection{4,6-二着基-2-丙硫基嘧啶(2)的合成}

搅拌下将 $\mathrm{NaOH}$ 固体 $27.8 \mathrm{~g}(0.7 \mathrm{~mol})$ 缓慢地加入 $200 \mathrm{~mL}$ 水中. 待其完全溶解, 整个反应体系冷却至室温 后, 补加 $100 \mathrm{~mL}$ 水和 $100 \mathrm{~mL}$ 乙醇. 将 2-硫代巴比妥酸 $100.0 \mathrm{~g}$ (0.7 mol)缓慢加入反应体系中, 待完全溶解后, 滴加碘代正丙烷 $68 \mathrm{~mL}(0.7 \mathrm{~mol})$. 室温下反应 $72 \mathrm{~h}$, 反 应体系中有大量絮状沉淀生成. 减压蒸除体系中的大部 分溶剂, 用 $4 \mathrm{~mol} / \mathrm{L}$ 稀盐酸调节 $\mathrm{pH}=3$. 减压抽滤, 并用 水和少量乙醇洗涤滤出物, 干燥后得到 $90.2 \mathrm{~g}$ 白色固体 $2^{[16]}$, 产率为 $70 \%$. m.p. $159 \sim 178{ }^{\circ} \mathrm{C}$ (文献值 ${ }^{[16]} 160 \sim 180$ $\left.{ }^{\circ} \mathrm{C}\right)$.

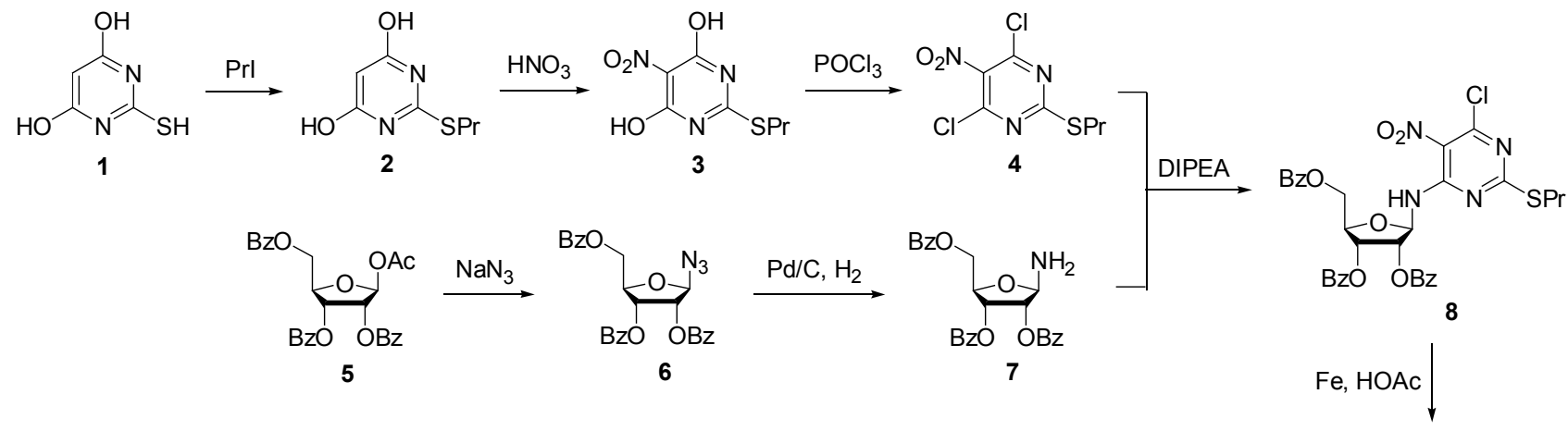

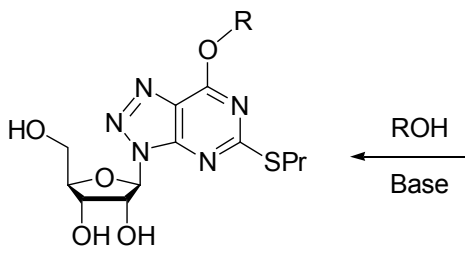

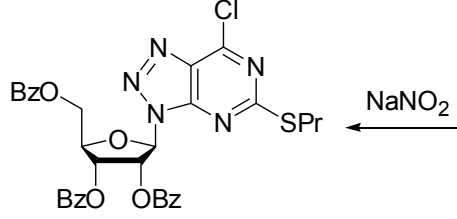

10

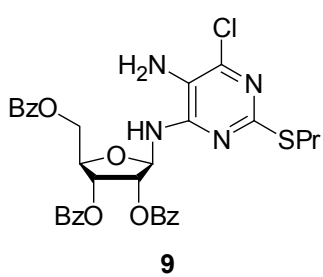

$\mathrm{R}=\mathrm{Me}, \mathrm{Et}$, $\mathrm{Pr}$, iso- $\mathrm{Pr}$, Bu, iso- $\mathrm{Bu}$, sec-Bu, $p-\mathrm{MeOC}_{6} \mathrm{H}_{4} \mathrm{CH}_{2} \mathrm{CH}_{2}, p-\mathrm{FC}_{6} \mathrm{H}_{4} \mathrm{CH}_{2} \mathrm{CH}_{2}, \pi \rrbracket-\mathrm{CH}_{2} \mathrm{CH}_{2}$

Scheme 2 


\subsubsection{5-硝基-4,6-二着基-2-丙硫基嘧啶(3)的合成}

称取 $20.0 \mathrm{~g}(0.1 \mathrm{~mol})$ 的化合物 2 , 取其中的一小部 分置于三口烧瓶中, 加入 $200 \mathrm{~mL}$ 冰醋酸使其溶解, 然 后再加入浓硝酸 $15 \mathrm{~mL}(0.3 \mathrm{~mol})$, 搅拌下缓慢升温至 50 ${ }^{\circ} \mathrm{C}$. 少量多次地向反应体系中加入剩下的化合物 2 , 控 制加料速度保持体系温度在 $50 \sim 60{ }^{\circ} \mathrm{C}$ 之间. 投料完毕 后控制温度在 $55{ }^{\circ} \mathrm{C}$ 左右, 反应 $1 \mathrm{~h}$ 后停止加热. 待体系 温度冷却至室温后, 将反应液缓慢倒入足量的冰水混合 物中, 有大量的沉淀生成. 减压抽滤, 并用大量的水冲 洗滤饼得到 $17.4 \mathrm{~g}$ 淡黄色固体 3, 产率为 70\%. m.p. $197 \sim 198{ }^{\circ} \mathrm{C}$ (文献值 ${ }^{[16]} 197 \sim 198{ }^{\circ} \mathrm{C}$ ).

\subsubsection{5-硝基-2-丙硫基-4,6-二氯嘧啶(4)的合成}

称取 $5.0 \mathrm{~g}$ (21.6 mmol)化合物 3 置于三口烧瓶中, 加入三氯氧磷 $17 \mathrm{~mL}(182.9 \mathrm{mmol})$ 使其溶解. 缓慢滴加 $N, N$-二甲基苯胺 $6.0 \mathrm{~mL}(47.5 \mathrm{mmol})$, 滴加完毕后升温 至回流. 保持回流反应 $1 \mathrm{~h}$ 后停止加热, 体系冷却至室 温. 将反应液缓慢滴加入大量冰水混合物中, 控制体系 温度在 $10{ }^{\circ} \mathrm{C}$ 以下. 滴加完毕后搅拌 $30 \mathrm{~min}$, 充分水解掉 过量的三氯氧磷. 用二氯甲烷 $(15 \mathrm{~mL} \times 3)$ 对水解液进行 萃取, 合并有机相. 经无水硫酸钠干燥, 过滤, 减压蒸 除溶剂后, 粗产物经柱色谱(二氯甲烷/石油醚, $V: V=$ $1: 8$ )分离提纯后得到 $5.5 \mathrm{~g}$ 黄色液体 $\mathbf{4}^{[17]}$, 产率为 $94 \%$. 1.2.4 1-叠氮基- $2,3,5$-三- $O$-苯甲酰基- $\beta$ - $D$-呋喃核糖 (6) 的合成

称取 $10.0 \mathrm{~g}(19.8 \mathrm{mmol})$ 化合物 5 置于单口烧瓶中, 加入 $100 \mathrm{~mL}$ 二氯甲烷将其溶解后, 加入 $1.5 \mathrm{~g}(22.6$ mmol) $\mathrm{NaN}_{3}$ 和无水四氯化锡 $4 \mathrm{~mL}$ (34.2 mmol), 常温下 搅拌反应. TLC 监测反应(乙酸乙酯/石油醚, $V: V=1$ : 10), 反应结束后, 向体系中加入 $40 \mathrm{~mL}$ 水, 用 $2 \mathrm{~mol} / \mathrm{L}$ 的 $\mathrm{NaOH}$ 调节 $\mathrm{pH}$ 至中性. 用二氯甲烷 $(15 \mathrm{~mL} \times 3)$ 对反 应液进行萃取, 合并有机相. 经无水硫酸钠干燥, 过滤, 减压蒸除溶剂后得到无色粘稠产物. 粗产物经柱色谱 (乙酸乙酯/石油醚, $V: V=1: 10$ )分离提纯后得到 $8.2 \mathrm{~g}$ 蜡状白色固体 $\mathbf{6}^{[18]}$, 产率为 $85 \%$.

\section{2 .54 - $\left[\left(2^{\prime}, 3^{\prime}, 5^{\prime}\right.\right.$-三- $O$-苯甲酰基)- $\beta$ - $D$-呋喃核糖基] 氨} 基-5-硝基-2-丙硫基-6-氯嘧啶(8)的合成

称取 $20.0 \mathrm{~g}$ (41.0 mmol)化合物 6 置于三口烧瓶中, 加入 $250 \mathrm{~mL}$ 乙酸乙酯将其溶解后, 加入 $0.2 \mathrm{~g} \mathrm{Pd} / \mathrm{C}$, 并 向体系中通入氢气, 室温下搅拌反应 $12 \mathrm{~h}$. 由于生成的 化合物 7 在室温下非常不稳定, 所以无需分离, 直接进 行下一步反应.

称取 $11.0 \mathrm{~g}$ (41.0 mmol)化合物 4 置于三口烧瓶中, 加入 $20 \mathrm{~mL}$ 四氢呋喃与 $11 \mathrm{~mL}(63.0 \mathrm{mmol}) \mathrm{N}, N$-二异丙 基乙胺, 常温下摚拌反应. 将化合物 7 通过加有硅藻土 及无水硫酸钠的滴液漏斗加入反应体系, 油浴条件下升
温至 $40{ }^{\circ} \mathrm{C}$, 反应 $5 \mathrm{~h}$. 反应完成后减压蒸除溶剂, 粗产 物经柱色谱(乙酸乙酯/石油醚, $V: V=1: 8$ )分离提纯后 得到 $15.4 \mathrm{~g}$ 浅黄色固体 $\mathbf{8}$, 产率为 $54 \%$, m.p. $138 \sim 140$ ${ }^{\circ} \mathrm{C}$; ${ }^{1} \mathrm{H}$ NMR (400 MHz, $\left.\mathrm{CDCl}_{3}\right) \delta: 8.35(\mathrm{~d}, J=7.4 \mathrm{~Hz}, 1 \mathrm{H}$, $\mathrm{NH}), 8.10 \sim 7.95(\mathrm{~m}, 6 \mathrm{H}, o-\mathrm{ArH}), 7.59 \sim 7.54(\mathrm{~m}, 3 \mathrm{H}$, $p$-ArH), $7.47 \sim 7.35$ (m, 6H, $m$-ArH), $6.32(\mathrm{dd}, J=7.4,4.2$ $\left.\mathrm{Hz}, 1 \mathrm{H}, \mathrm{H}-3^{\prime}\right), 5.88 \sim 5.84$ (m, 2H, H-1', H-2'), 4.74 4.62 $\left(\mathrm{m}, 3 \mathrm{H}, \mathrm{H}-4^{\prime}, \mathrm{H}^{-} 5^{\prime} \alpha, \mathrm{H}-5^{\prime} \beta\right), 3.10 \sim 2.97(\mathrm{~m}, 2 \mathrm{H}$, $\mathrm{SCH}_{2} \mathrm{CH}_{2}-\mathrm{CH}_{3}$ ), 1.68 (sextet, $J=7.2 \mathrm{~Hz}, 2 \mathrm{H}, \mathrm{SCH}_{2} \mathrm{CH}_{2}-$ $\left.\mathrm{CH}_{3}\right), 0.94\left(\mathrm{t}, J=7.3 \mathrm{~Hz}, 3 \mathrm{H}, \mathrm{SCH}_{2} \mathrm{CH}_{2} \mathrm{CH}_{3}\right) ;{ }^{13} \mathrm{C} \mathrm{NMR}$ $\left(100 \mathrm{MHz}, \mathrm{CDCl}_{3}\right) \delta: 174.56,166.20,165.31,165.25$, $155.07,154.72,133.81,133.71,133.47,129.89,129.78$, $129.72,129.32,128.65,128.56,128.54,124.09,84.93$, $79.70,74.63,71.62,63.74,33.73,22.20,13.38$; IR (KBr) $v: 3429,2965,1727,1565,1511,1326,1268,1227,1112$, 1069, $710 \mathrm{~cm}^{-1}$; HRMS (ESI) calcd for $\mathrm{C}_{33} \mathrm{H}_{30} \mathrm{ClN}_{4} \mathrm{O}_{9} \mathrm{~S}$ $[\mathrm{M}+\mathrm{H}]^{+}$693.1422, found 693.1407.

1.2.65-氨基-4-[( $2^{\prime}, 3^{\prime}, 5^{\prime}$-三- $O$ - 苯甲酰基)- $\beta$ - $D$-呋喃核 糖基]氨基-2-丙硫基-6-氯嘧啶(9)的合成

称取 $15.4 \mathrm{~g}$ (22.2 mmol)化合物 8 溶于 $200 \mathrm{~mL}$ 冰醋 酸中, 加入 $10.0 \mathrm{~g}(179.1 \mathrm{mmol})$ 还原铁粉, 常温下摚拌 反应 $48 \mathrm{~h}$. 加水后, 用乙酸乙酯 $(15 \mathrm{~mL} \times 3)$ 对反应液进 行萃取, 合并有机相. 经无水硫酸钠干燥, 过滤, 减压 蒸除溶剂后得到粗产物. 粗产物经柱色谱 (乙酸乙酯/ 石油醚, $V: V=1: 5$ )分离提纯后得到 $13.0 \mathrm{~g}$ 黄色粘稠状 液体 $\mathbf{9}^{[12]}$, 产率为 $88 \%$.

1.2.79-[(2', 3', $5^{\prime}-$ 三- $O$ - 苯甲酰基 $)-\beta-D$ - 呋喃核糖 基]-2-丙硫基-6-氯-8-氮杂嘌呤(10)的合成

称取 $13.3 \mathrm{~g}$ (20.0 mmol)化合物 9 溶于 $200 \mathrm{~mL}$ 冰醋 酸中. 向反应体系中加入 $20 \mathrm{~mL}$ 水, 在冰盐浴条件下将 反应体系温度降至 $0{ }^{\circ} \mathrm{C}$. 将 $1.6 \mathrm{~g}(23.2 \mathrm{mmol})$ 亚硝酸钠 溶于 $5 \mathrm{~mL}$ 水中, 缓慢滴加入反应体系, 滴加过程控制 反应体系温度在 $5{ }^{\circ} \mathrm{C}$ 以下. 滴加完毕后, 在 $5{ }^{\circ} \mathrm{C}$ 以下反 应 $1 \mathrm{~h}$, 然后转移至 $50{ }^{\circ} \mathrm{C}$ 的恒温水浴中继续反应 $1.5 \mathrm{~h}$. 加水后, 用二氯甲烷 $(15 \mathrm{~mL} \times 3)$ 对反应液进行萃取, 合 并有机相. 经无水硫酸钠干燥, 过滤, 减压蒸除溶剂后 得到粗产物. 粗产物经柱色谱(乙酸乙酯/石油醚, $V$ : $V=1: 8$ ) 分离提纯后得到 $11.5 \mathrm{~g}$ 淡黄色固体 10, 产率为 85\%. m.p. 122 124 ${ }^{\circ} \mathrm{C} ;{ }^{1} \mathrm{H}$ NMR (400 MHz, $\left.\mathrm{CDCl}_{3}\right) \delta$ : $7.99 \sim 7.97(\mathrm{~m}, 6 \mathrm{H}, o-\mathrm{ArH}), 7.60 \sim 7.53$ (m, 3H, $p-\mathrm{ArH})$, $7.42 \sim 7.37$ (m, 6H, m-ArH), 6.79 (d, $\left.J=2.5 \mathrm{~Hz}, 1 \mathrm{H}, \mathrm{H}-1^{\prime}\right)$, 6.55 (q, $\left.J=2.8 \mathrm{~Hz}, 1 \mathrm{H}, \mathrm{H}-2^{\prime}\right), 6.34(\mathrm{t}, J=6.0 \mathrm{~Hz}, 1 \mathrm{H}$, H-3'), 4.93 (q, $\left.J=4.8 \mathrm{~Hz}, 1 \mathrm{H}, \mathrm{H}-4^{\prime}\right), 4.82 \sim 4.78(\mathrm{~m}, 1 \mathrm{H}$, $\left.\mathrm{H}-5^{\prime} \alpha\right), 4.66 \sim 4.62\left(\mathrm{~m}, 1 \mathrm{H}, \mathrm{H}-5^{\prime} \beta\right), 3.30 \sim 3.20(\mathrm{~m}, 2 \mathrm{H}$, $\mathrm{SCH}_{2} \mathrm{CH}_{2} \mathrm{CH}_{3}$ ), 1.80 (sextet, $J=7.3 \mathrm{~Hz}, 2 \mathrm{H}, \mathrm{SCH}_{2^{-}}$ 
$\left.\mathrm{CH}_{2} \mathrm{CH}_{3}\right), 1.05\left(\mathrm{t}, J=7.3 \mathrm{~Hz}, 3 \mathrm{H}, \mathrm{SCH}_{2} \mathrm{CH}_{2} \mathrm{CH}_{3}\right) ;{ }^{13} \mathrm{C}$ NMR $\left(100 \mathrm{MHz}, \mathrm{CDCl}_{3}\right) \delta: 173.11,166.07,165.11$, $165.03,153.39,150.62,133.93,133.73,133.31,132.06$, $129.87,129.83,129.71,129.24,128.62,128.55,128.44$, $88.42,80.69,74.19,71.46,63.26,33.87,21.89,13.41$; IR (KBr) v: 3435, 3061, 2961, 2875, 1723, 1711, 1587, 1554, 1452, 1268, 1141, 1128, 1093, 1068, 979, $710 \mathrm{~cm}^{-1}$; HRMS (ESI) calcd for $\mathrm{C}_{33} \mathrm{H}_{28} \mathrm{ClN}_{5} \mathrm{NaO}_{7} \mathrm{~S}[\mathrm{M}+\mathrm{Na}]^{+}$ 696.1296, found 696.1290.

1.2.86-烷氧基-2-丙硫基-8-氮杂嘌呤核甘 $(\mathbf{1 1 a} \sim \mathbf{1 1} \mathbf{j})$ 的合成

合成方法 A: 将 $0.04 \mathrm{~g}(1.9 \mathrm{mmol})$ 金属钠丝加入到 $15 \mathrm{~mL}$ 甲醇中, 适当加热形成甲醇钠-甲醇的溶液体系. 将 $0.5 \mathrm{~g}$ (0.7 mmol)化合物 $\mathbf{1 0}$ 溶于甲醇中, 加入制备好 的甲醇钠-甲醇的溶液, 室温下反应 $4 \mathrm{~h}$. 减压蒸除溶剂, 向得到的粗产物中加入 $20 \mathrm{~mL}$ 水, 用冰醋酸调节 $\mathrm{pH}=$ 6. 用乙酸乙酯 $(15 \mathrm{~mL} \times 3)$ 进行萃取, 合并有机相. 经无 水硫酸钠干燥, 过滤, 减压蒸除溶剂后得到粗产物. 粗 产物经柱色谱(乙酸乙酯/石油醚, $V: V=2: 1$ )分离提 纯, 乙酸乙酯与石油醚重结晶得到化合物 $11 \mathrm{a}$.

参照上述方法分别用乙醇、正丙醇、异丙醇、正丁 醇、异丁醇、仲丁醇代替甲醇制得化合物 11b 11g.

6-甲氧基-2-丙硫基-8-氮杂嘌呤核苷(11a)：白色固 体, 产率为 39\%. m.p. $103 \sim 105{ }^{\circ} \mathrm{C} ;{ }^{1} \mathrm{H}$ NMR $(400 \mathrm{MHz}$, DMSO- $\left.d_{6}\right) \delta: 6.21\left(\mathrm{~d}, J=4.8 \mathrm{~Hz}, 1 \mathrm{H}, \mathrm{H}-1^{\prime}\right), 5.66$ (d, $J=$ $\left.5.8 \mathrm{~Hz}, 1 \mathrm{H}, \mathrm{OH}-2^{\prime}\right), 5.35$ (d, $J=5.6 \mathrm{~Hz}, 1 \mathrm{H}, \mathrm{OH}-3$ '), 4.91 (q, $\left.J=5.1 \mathrm{~Hz}, 1 \mathrm{H}, \mathrm{H}-2^{\prime}\right), 4.83$ (t, $\left.J=5.8 \mathrm{~Hz}, 1 \mathrm{H}, \mathrm{OH}-5^{\prime}\right)$, 4.38 (q, $J=5.1 \mathrm{~Hz}, 1 \mathrm{H}, \mathrm{H}-3$ '), 4.20 (s, 3H, $\mathrm{OCH}_{3}$ ), 4.05 (q, $J=4.8 \mathrm{~Hz}, 1 \mathrm{H}, \mathrm{H}-4$ '), $3.69 \sim 3.64\left(\mathrm{~m}, 1 \mathrm{H}, \mathrm{H}-5^{\prime} \alpha\right), 3.56 \sim$ $3.50\left(\mathrm{~m}, 1 \mathrm{H}, \mathrm{H}-5^{\prime} \beta\right), 3.27 \sim 3.16\left(\mathrm{~m}, 2 \mathrm{H}, \mathrm{SCH}_{2} \mathrm{CH}_{2} \mathrm{CH}_{3}\right)$, 1.78 (sextet, $J=7.2 \mathrm{~Hz}, 2 \mathrm{H}, \mathrm{SCH}_{2} \mathrm{CH}_{2} \mathrm{CH}_{3}$ ), 1.05 (t, $J=$ $\left.7.4 \mathrm{~Hz}, 3 \mathrm{H}, \mathrm{SCH}_{2} \mathrm{CH}_{2} \mathrm{CH}_{3}\right) ;{ }^{13} \mathrm{C}$ NMR (100 MHz, DMSO$\left.d_{6}\right) \delta: 170.43,160.14,151.35,123.63,89.67,85.99,72.82$, 70.56, 61.75, 55.04, 32.66, 22.08, 13.18; IR (KBr) v: 3421, 3264, 2965, 2942, 2872, 1593, 1483, 1451, 1395, 1335, 1283, 1230, 1204, 1175, 1082, $1037 \mathrm{~cm}^{-1}$; HRMS (ESI) calcd for $\mathrm{C}_{13} \mathrm{H}_{20} \mathrm{~N}_{5} \mathrm{O}_{5} \mathrm{~S}[\mathrm{M}+\mathrm{H}]{ }^{+} 358.1185$, found 358.1183 .

6-乙氧基-2-丙硫基-8-氮杂嘌呤核苷(11b): 白色固 体, 产率为 $41 \%$. m.p. $160 \sim 161{ }^{\circ} \mathrm{C} ;{ }^{1} \mathrm{H}$ NMR $(400 \mathrm{MHz}$, DMSO- $\left.d_{6}\right) \delta: 6.17\left(\mathrm{~d}, J=4.8 \mathrm{~Hz}, 1 \mathrm{H}, \mathrm{H}-1^{\prime}\right), 5.61$ (d, $J=$ $\left.5.8 \mathrm{~Hz}, 1 \mathrm{H}, \mathrm{OH}-2^{\prime}\right), 5.31$ (d, $J=5.6 \mathrm{~Hz}, 1 \mathrm{H}, \mathrm{OH}-3$ '), 4.87 (q, $\left.J=5.2 \mathrm{~Hz}, 1 \mathrm{H}, \mathrm{H}-2^{\prime}\right), 4.79$ (t, $\left.J=5.6 \mathrm{~Hz}, 1 \mathrm{H}, \mathrm{OH}-5^{\prime}\right)$, 4.66 (q, $J=7.0 \mathrm{~Hz}, 2 \mathrm{H}, \mathrm{OCH}_{2} \mathrm{CH}_{3}$ ), 4.33 (q, $J=4.9 \mathrm{~Hz}$, $\left.1 \mathrm{H}, \mathrm{H}-3^{\prime}\right), 4.01$ (q, $J=4.9 \mathrm{~Hz}, 1 \mathrm{H}, \mathrm{H}-4$ '), $3.65 \sim 3.59$ (m, $\left.1 \mathrm{H}, \mathrm{H}-5^{\prime} \alpha\right), 3.51 \sim 3.46\left(\mathrm{~m}, 1 \mathrm{H}, \mathrm{H}-5^{\prime} \beta\right), 3.24 \sim 3.12(\mathrm{~m}, 2 \mathrm{H}$, $\mathrm{SCH}_{2} \mathrm{CH}_{2} \mathrm{CH}_{3}$ ), 1.75 (sextet, $J=7.2 \mathrm{~Hz}, 2 \mathrm{H}, \mathrm{SCH}_{2} \mathrm{CH}_{2}-$ $\left.\mathrm{CH}_{3}\right), 1.45$ (t, $\left.J=7.0 \mathrm{~Hz}, 3 \mathrm{H}, \mathrm{OCH}_{2} \mathrm{CH}_{3}\right), 1.02$ (t, $J=7.4$ $\left.\mathrm{Hz}, 3 \mathrm{H}, \mathrm{SCH}_{2} \mathrm{CH}_{2} \mathrm{CH}_{3}\right) ;{ }^{13} \mathrm{C}$ NMR (100 MHz, DMSO- $\left.d_{6}\right)$ $\delta: 170.38,159.78,151.41,123.64,89.65,85.99,72.77$, $70.55,64.16,61.74,32.64,22.14,14.05,13.19$; IR (KBr) v: 3442, 3310, 2971, 2941, 2869, 2831, 1586, 1486, 1435, 1379, 1342, 1263, 1200, 1051, $1010 \mathrm{~cm}^{-1}$; HRMS (ESI) calcd for $\mathrm{C}_{14} \mathrm{H}_{22} \mathrm{~N}_{5} \mathrm{O}_{5} \mathrm{~S}[\mathrm{M}+\mathrm{H}]^{+}$372.1342, found 372.1349 .

6-正丙氧基-2-丙硫基-8-氮杂嘌呤核苷(11c): 白色 固体, 产率为 39\%. m.p. 82 84 ${ }^{\circ} \mathrm{C} ;{ }^{1} \mathrm{H}$ NMR (400 MHz, DMSO- $\left.d_{6}\right) \delta: 6.17\left(\mathrm{~d}, J=4.7 \mathrm{~Hz}, 1 \mathrm{H}, \mathrm{H}-1^{\prime}\right), 5.61$ (d, $J=$ $\left.5.8 \mathrm{~Hz}, 1 \mathrm{H}, \mathrm{OH}-2^{\prime}\right), 5.31$ (d, $J=5.5 \mathrm{~Hz}, 1 \mathrm{H}, \mathrm{OH}-3$ '), 4.87 (q, $\left.J=5.0 \mathrm{~Hz}, 1 \mathrm{H}, \mathrm{H}-2^{\prime}\right), 4.79$ (t, $\left.J=5.7 \mathrm{~Hz}, 1 \mathrm{H}, \mathrm{OH}-5^{\prime}\right)$, 4.57 (t, $J=6.0 \mathrm{~Hz}, 2 \mathrm{H}, \mathrm{OCH}_{2} \mathrm{CH}_{2} \mathrm{CH}_{3}$ ), 4.33 (q, $J=4.9$ Hz, 1H, H-3'), 4.01 (q, J=4.9 Hz, 1H, H-4'), 3.65 3.59 $\left(\mathrm{m}, 1 \mathrm{H}, \mathrm{H}-5^{\prime} \alpha\right), 3.51 \sim 3.46\left(\mathrm{~m}, 1 \mathrm{H}, \mathrm{H}-5^{\prime} \beta\right), 3.24 \sim 3.12(\mathrm{~m}$, $2 \mathrm{H}, \mathrm{SCH}_{2} \mathrm{CH}_{2} \mathrm{CH}_{3}$ ), 1.86 (sextet, $J=7.1 \mathrm{~Hz}, 2 \mathrm{H}, \mathrm{OCH}_{2}-$ $\mathrm{CH}_{2} \mathrm{CH}_{3}$ ), 1.75 (sextet, $J=7.3 \mathrm{~Hz}, 2 \mathrm{H}, \mathrm{SCH}_{2} \mathrm{CH}_{2} \mathrm{CH}_{3}$ ), $1.04 \sim 1.00\left(\mathrm{~m}, 6 \mathrm{H}, \mathrm{SCH}_{2} \mathrm{CH}_{2} \mathrm{CH}_{3}, \mathrm{OCH}_{2} \mathrm{CH}_{2} \mathrm{CH}_{3}\right) ;{ }^{13} \mathrm{C}$ NMR (100 MHz, DMSO- $\left.d_{6}\right) \delta: 170.36,159.93,151.43$, $123.62,89.66,85.99,72.78,70.55,69.44,61.74,32.65$, $22.15,21.45,13.20,10.11$; IR (KBr) v: 3372, 2959, 2926, 2869, 2846, 1587, 1442, 1374, 1335, 1280, 1191, 1076 $\mathrm{cm}^{-1}$; HRMS (ESI) calcd for $\mathrm{C}_{15} \mathrm{H}_{24} \mathrm{~N}_{5} \mathrm{O}_{5} \mathrm{~S}[\mathrm{M}+\mathrm{H}]^{+}$ 386.1498, found 386.1493.

6-异丙氧基-2-丙硫基-8-氮杂嘌呤核苷(11d): 白色 固体, 产率为 $35 \%$. m.p. $129 \sim 130{ }^{\circ} \mathrm{C} ;{ }^{1} \mathrm{H}$ NMR (400 $\left.\mathrm{MHz}, \mathrm{DMSO}-d_{6}\right) \delta: 6.17(\mathrm{~d}, J=4.9 \mathrm{~Hz}, 1 \mathrm{H}, \mathrm{H}-1$ '), $5.65 \sim$ $5.59\left[\mathrm{~m}, 2 \mathrm{H}, \mathrm{OH}-2^{\prime}, \mathrm{OCH}\left(\mathrm{CH}_{3}\right)_{2}\right], 5.31(\mathrm{~d}, J=5.6 \mathrm{~Hz}, 1 \mathrm{H}$, OH-3'), 4.87 (q, $\left.J=5.1 \mathrm{~Hz}, 1 \mathrm{H}, \mathrm{H}-2^{\prime}\right), 4.79$ (t, $J=5.8 \mathrm{~Hz}$, $\left.1 \mathrm{H}, \mathrm{OH}-5^{\prime}\right), 4.33$ (q, $\left.J=4.9 \mathrm{~Hz}, 1 \mathrm{H}, \mathrm{H}-3^{\prime}\right), 4.01$ (q, $J=4.9$ $\left.\mathrm{Hz}, 1 \mathrm{H}, \mathrm{H}-4^{\prime}\right), 3.65 \sim 3.59\left(\mathrm{~m}, 1 \mathrm{H}, \mathrm{H}-5^{\prime} \alpha\right), 3.51 \sim 3.46(\mathrm{~m}$, $1 \mathrm{H}, \mathrm{H}-5$ ' $\beta$ ), $3.23 \sim 3.12\left(\mathrm{~m}, 2 \mathrm{H}, \mathrm{SCH}_{2} \mathrm{CH}_{2} \mathrm{CH}_{3}\right.$ ), 1.75 (sextet, $\left.J=7.4 \mathrm{~Hz}, 2 \mathrm{H}, \mathrm{SCH}_{2} \mathrm{CH}_{2} \mathrm{CH}_{3}\right), 1.45[\mathrm{~d}, J=6.2 \mathrm{~Hz}$, $\left.6 \mathrm{H}, \mathrm{OCH}\left(\mathrm{CH}_{3}\right)_{2}\right], 1.02$ (t, $\left.J=7.3 \mathrm{~Hz}, 3 \mathrm{H}, \mathrm{SCH}_{2} \mathrm{CH}_{2} \mathrm{CH}_{3}\right)$; ${ }^{13} \mathrm{C}$ NMR (100 MHz, DMSO- $\left.d_{6}\right) \quad \delta: 170.34,159.40$, $151.49,123.79,89.66,85.99,72.76,71.96,70.55,61.74$, 32.65, 22.24, 21.45, 13.20; IR (KBr) v: 3369, 2959, 2928, 2872, 1585, 1441, 1384, 1329, 1282, 1196, $1077 \mathrm{~cm}^{-1}$; HRMS (ESI) calcd for $\mathrm{C}_{15} \mathrm{H}_{24} \mathrm{~N}_{5} \mathrm{O}_{5} \mathrm{~S}[\mathrm{M}+\mathrm{H}]^{+} 386.1498$, found 386.1506 .

6-正丁氧基-2-丙硫基-8-氮杂嘌呤核苷(11e): 白色 固体, 产率为 $38 \%$. m.p. $76 \sim 78{ }^{\circ} \mathrm{C} ;{ }^{1} \mathrm{H}$ NMR (400 MHz, 
DMSO- $\left.d_{6}\right) \delta: 6.17$ (d, $\left.J=4.8 \mathrm{~Hz}, 1 \mathrm{H}, \mathrm{H}-1^{\prime}\right), 5.61$ (d, $J=$ $\left.5.9 \mathrm{~Hz}, 1 \mathrm{H}, \mathrm{OH}-2^{\prime}\right), 5.31$ (d, $\left.J=5.5 \mathrm{~Hz}, 1 \mathrm{H}, \mathrm{OH}-3^{\prime}\right), 4.87$ (q, $\left.J=5.1 \mathrm{~Hz}, 1 \mathrm{H}, \mathrm{H}-2^{\prime}\right), 4.79$ (t, $\left.J=5.7 \mathrm{~Hz}, 1 \mathrm{H}, \mathrm{OH}-5^{\prime}\right)$, 4.62 (t, $\left.J=6.7 \mathrm{~Hz}, 2 \mathrm{H}, \mathrm{OCH}_{2} \mathrm{CH}_{2} \mathrm{CH}_{2} \mathrm{CH}_{3}\right), 4.33$ (q, $J=$ $\left.5.0 \mathrm{~Hz}, 1 \mathrm{H}, \mathrm{H}-3^{\prime}\right), 4.01$ (q, $\left.J=4.9 \mathrm{~Hz}, 1 \mathrm{H}, \mathrm{H}-4^{\prime}\right), 3.65 \sim$ $3.59\left(\mathrm{~m}, 1 \mathrm{H}, \mathrm{H}-5^{\prime} \alpha\right), 3.51 \sim 3.46\left(\mathrm{~m}, 1 \mathrm{H}, \mathrm{H}-5^{\prime} \beta\right), 3.24 \sim$ $3.12\left(\mathrm{~m}, 2 \mathrm{H}, \mathrm{SCH}_{2} \mathrm{CH}_{2} \mathrm{CH}_{3}\right), 1.86 \sim 1.71\left(\mathrm{~m}, 4 \mathrm{H}, \mathrm{OCH}_{2}-\right.$ $\mathrm{CH}_{2} \mathrm{CH}_{2} \mathrm{CH}_{3}, \mathrm{SCH}_{2} \mathrm{CH}_{2} \mathrm{CH}_{3}$ ), 1.46 (sextet, $J=7.5 \mathrm{~Hz}, 2 \mathrm{H}$, $\mathrm{OCH}_{2} \mathrm{CH}_{2} \mathrm{CH}_{2} \mathrm{CH}_{3}$ ), 1.02 (t, $J=7.3 \mathrm{~Hz}, 3 \mathrm{H}, \mathrm{SCH}_{2} \mathrm{CH}_{2}-$ $\left.\mathrm{CH}_{3}\right), 0.96\left(\mathrm{t}, J=7.4 \mathrm{~Hz}, 3 \mathrm{H}, \mathrm{OCH}_{2} \mathrm{CH}_{2} \mathrm{CH}_{2} \mathrm{CH}_{3}\right) ;{ }^{13} \mathrm{C}$ NMR $\left(100 \mathrm{MHz}\right.$, DMSO- $\left.d_{6}\right) \delta: 170.36,159.92,151.43$, $123.63,89.66,85.99,72.78,70.55,67.73,61.74,32.65$, $30.03,22.15,18.54,13.54,13.19$; IR (KBr) v: 3392, 2961, 2929, 2869, 1587, 1443, 1375, 1334, 1282, 1192, 1077 $\mathrm{cm}^{-1}$; HRMS (ESI) calcd for $\mathrm{C}_{16} \mathrm{H}_{26} \mathrm{~N}_{5} \mathrm{O}_{5} \mathrm{~S}[\mathrm{M}+\mathrm{H}]^{+}$ 400.1655 , found 400.1652 .

6-异丁氧基-2-丙硫基-8-氮杂嘌呤核苷(11f): 白色 固体, 产率为 $25 \%$. m.p. $72 \sim 74{ }^{\circ} \mathrm{C} ;{ }^{1} \mathrm{H}$ NMR $(400 \mathrm{MHz}$, DMSO- $\left.d_{6}\right) \delta: 6.17\left(\mathrm{~d}, J=4.8 \mathrm{~Hz}, 1 \mathrm{H}, \mathrm{H}-1^{\prime}\right), 5.61$ (d, $J=$ $\left.5.8 \mathrm{~Hz}, 1 \mathrm{H}, \mathrm{OH}-2^{\prime}\right), 5.31$ (d, $\left.J=5.6 \mathrm{~Hz}, 1 \mathrm{H}, \mathrm{OH}-3^{\prime}\right), 4.87$ (q, $\left.J=5.0 \mathrm{~Hz}, 1 \mathrm{H}, \mathrm{H}-2^{\prime}\right), 4.78\left(\mathrm{t}, J=5.7 \mathrm{~Hz}, 1 \mathrm{H}, \mathrm{OH}-5^{\prime}\right)$, $4.41\left[\mathrm{~d}, J=6.7 \mathrm{~Hz}, 2 \mathrm{H}, \mathrm{OCH}_{2} \mathrm{CH}\left(\mathrm{CH}_{3}\right)_{2}\right], 4.34$ (q, $J=5.0$ $\mathrm{Hz}, 1 \mathrm{H}, \mathrm{H}-3^{\prime}$ ), 4.01 (q, $\left.J=5.0 \mathrm{~Hz}, 1 \mathrm{H}, \mathrm{H}-4^{\prime}\right), 3.65 \sim 3.59$ (m, $\left.1 \mathrm{H}, \mathrm{H}-5^{\prime} \alpha\right), 3.51 \sim 3.45\left(\mathrm{~m}, 1 \mathrm{H}, \mathrm{H}-5^{\prime} \beta\right), 3.23 \sim 3.12(\mathrm{~m}$, $2 \mathrm{H}, \mathrm{SCH}_{2} \mathrm{CH}_{2} \mathrm{CH}_{3}$ ), 2.18 [heptet, $J=6.7 \mathrm{~Hz}, 1 \mathrm{H}, \mathrm{OCH}_{2}$ $\left.\mathrm{CH}\left(\mathrm{CH}_{3}\right)_{2}\right], 1.76$ (sextet, $J=7.3 \mathrm{~Hz}, 2 \mathrm{H}, \mathrm{SCH}_{2} \mathrm{CH}_{2} \mathrm{CH}_{3}$ ), $1.04 \sim 0.99\left[\mathrm{~m}, 9 \mathrm{H}, \mathrm{SCH}_{2} \mathrm{CH}_{2} \mathrm{CH}_{3}, \mathrm{OCH}_{2} \mathrm{CH}\left(\mathrm{CH}_{3}\right)_{2}\right] ;{ }^{13} \mathrm{C}$ NMR $\left(100 \mathrm{MHz}\right.$, DMSO- $\left.d_{6}\right) \delta: 170.34,159.99,151.45$, $123.59,89.67,85.98,73.60,72.78,70.55,61.74,32.64$, 27.27, 22.15, 18.75, 13.20; IR (KBr) v: 3340, 2962, 2926, 2873, 1587, 1442, 1388, 1333, 1288, 1194, $1080 \mathrm{~cm}^{-1}$; HRMS (ESI) calcd for $\mathrm{C}_{16} \mathrm{H}_{26} \mathrm{~N}_{5} \mathrm{O}_{5} \mathrm{~S}[\mathrm{M}+\mathrm{H}]^{+} 400.1655$, found 400.1654 .

6-仲丁氧基-2-丙硫基-8-氮杂嘌呤核苷(11g): 白色 固体, 产率为 $30 \%$. m.p. $98 \sim 100{ }^{\circ} \mathrm{C} ;{ }^{1} \mathrm{H}$ NMR $(400 \mathrm{MHz}$, DMSO- $\left.d_{6}\right) \delta: 6.16\left(\mathrm{~d}, J=4.8 \mathrm{~Hz}, 1 \mathrm{H}, \mathrm{H}-1^{\prime}\right), 5.61$ (d, $J=$ $5.8 \mathrm{~Hz}, 1 \mathrm{H}, \mathrm{OH}-2^{\prime}$ ), 5.47 [sextet, $J=6.2 \mathrm{~Hz}, 1 \mathrm{H}$, $\mathrm{OCH}\left(\mathrm{CH}_{3}\right) \mathrm{CH}_{2} \mathrm{CH}_{3}$ ], 5.31 (d, $\left.J=5.6 \mathrm{~Hz}, 1 \mathrm{H}, \mathrm{OH}-3^{\prime}\right), 4.87$ (q, $\left.J=5.1 \mathrm{~Hz}, 1 \mathrm{H}, \mathrm{H}-2^{\prime}\right), 4.78$ (t, $\left.J=5.7 \mathrm{~Hz}, 1 \mathrm{H}, \mathrm{OH}-5^{\prime}\right)$, 4.32 (q, $\left.J=4.9 \mathrm{~Hz}, 1 \mathrm{H}, \mathrm{H}-3^{\prime}\right), 4.00$ (q, $J=4.9 \mathrm{~Hz}, 1 \mathrm{H}$, H-4'), $3.65 \sim 3.59\left(\mathrm{~m}, 1 \mathrm{H}, \mathrm{H}-5^{\prime} \alpha\right), 3.51 \sim 3.45(\mathrm{~m}, 1 \mathrm{H}$, $\left.\mathrm{H}-5^{\prime} \beta\right), 3.23 \sim 3.11\left(\mathrm{~m}, 2 \mathrm{H}, \mathrm{SCH}_{2} \mathrm{CH}_{2} \mathrm{CH}_{3}\right), 1.86 \sim 1.73$ $\left[\mathrm{m}, 4 \mathrm{H}, \mathrm{SCH}_{2} \mathrm{CH}_{2} \mathrm{CH}_{3}, \mathrm{OCH}\left(\mathrm{CH}_{3}\right) \mathrm{CH}_{2} \mathrm{CH}_{3}\right], 1.41[\mathrm{~d}, J=$ $\left.6.2 \mathrm{~Hz}, 3 \mathrm{H}, \mathrm{OCH}\left(\mathrm{CH}_{3}\right) \mathrm{CH}_{2} \mathrm{CH}_{3}\right], 1.02$ (t, $J=7.3 \mathrm{~Hz}, 3 \mathrm{H}$, $\left.\mathrm{SCH}_{2} \mathrm{CH}_{2} \mathrm{CH}_{3}\right), 0.96\left[\mathrm{t}, J=7.4 \mathrm{~Hz}, 3 \mathrm{H}, \mathrm{OCH}\left(\mathrm{CH}_{3}\right) \mathrm{CH}_{2}-\right.$
$\left.\mathrm{CH}_{3}\right] ;{ }^{13} \mathrm{C}$ NMR $\left(100 \mathrm{MHz}\right.$, DMSO- $\left.d_{6}\right) \delta: 170.32,159.69$, $151.51,123.77,89.65,85.99,76.31,72.75,70.55,61.74$, $32.66,28.12,22.23,18.95,13.21,9.45$; IR (KBr) v: 3336, 2966, 2928, 2869, 1585, 1445, 1383, 1331, 1288, 1194, $1079 \mathrm{~cm}^{-1}$; HRMS (ESI) calcd for $\mathrm{C}_{16} \mathrm{H}_{26} \mathrm{~N}_{5} \mathrm{O}_{5} \mathrm{~S}[\mathrm{M}+\mathrm{H}]^{+}$ 400.1655 , found 400.1655 .

合成方法 $\mathrm{B}^{[19]}$ : 将 $130.0 \mathrm{mg}(0.9 \mathrm{mmol}) \mathrm{NaI}, 200.0$ $\mathrm{mg}(5.0 \mathrm{mmol}) \mathrm{NaOH}$ 和 $761.0 \mathrm{mg}(5.0 \mathrm{mmol}) 4$-甲氧基苯 乙醇溶于无水乙腈中, 室温下反应 $1 \mathrm{~h}$. 向反应体系中 加入 $600.0 \mathrm{mg}(0.9 \mathrm{mmol})$ 化合物 $\mathbf{1 0}$, 室温下反应 $48 \mathrm{~h}$. 减压蒸除溶剂, 向得到的粗产物中加入 $20 \mathrm{~mL}$ 水, 用冰 醋酸调节 $\mathrm{pH}=6$. 用乙酸乙酯 $(15 \mathrm{~mL} \times 3)$ 进行萃取, 合 并有机相. 经无水硫酸钠干燥, 过滤, 减压蒸除溶剂后 得到粗产物. 粗产物经柱色谱(乙酸乙酯/石油醚, $V$ : $V=2: 1$ )分离提纯, 乙酸乙酯与石油醚重结晶得到固体 $11 \mathrm{~h}$.

参照上述方法分别用 4-氟苯乙醇, 2-噻吩乙醇代替 4-甲氧基苯乙醇制得化合物 $\mathbf{1 1 i} \sim \mathbf{1 1}$ j.

6-(4-甲氧基苯乙氧基)-2-丙硫基-8-氮杂嘌呤核苷 (11h): 白色固体, 产率为 $26 \%$. m.p. $68 \sim 70{ }^{\circ} \mathrm{C} ;{ }^{1} \mathrm{H}$ NMR $\left(400 \mathrm{MHz}, \mathrm{DMSO}-d_{6}\right) \delta: 7.27 \sim 6.87(\mathrm{~m}, 4 \mathrm{H}, \mathrm{Ar}), 6.16(\mathrm{~d}$, $\left.J=4.7 \mathrm{~Hz}, 1 \mathrm{H}, \mathrm{H}-1^{\prime}\right), 5.61$ (d, $\left.J=5.7 \mathrm{~Hz}, 1 \mathrm{H}, \mathrm{OH}-2^{\prime}\right), 5.31$ (d, $\left.J=5.6 \mathrm{~Hz}, 1 \mathrm{H}, \mathrm{OH}-3^{\prime}\right), 4.86$ (q, $J=5.0 \mathrm{~Hz}, 1 \mathrm{H}, \mathrm{H}-2^{\prime}$ ), $4.80 \sim 4.76\left(\mathrm{~m}, 3 \mathrm{H}, \mathrm{OH}-5\right.$ ', $\left.\mathrm{OCH}_{2} \mathrm{CH}_{2}\right), 4.33$ (q, $J=4.7 \mathrm{~Hz}$, $\left.1 \mathrm{H}, \mathrm{H}-3^{\prime}\right), 4.01\left(\mathrm{q}, J=4.4 \mathrm{~Hz}, 1 \mathrm{H}, \mathrm{H}-4^{\prime}\right), 3.72(\mathrm{~s}, 3 \mathrm{H}$, $\left.\mathrm{OCH}_{3}\right), 3.65 \sim 3.59\left(\mathrm{~m}, 1 \mathrm{H}, \mathrm{H}-5^{\prime} \alpha\right), 3.51 \sim 3.45(\mathrm{~m}, 1 \mathrm{H}$, $\left.\mathrm{H}-5^{\prime} \beta\right), 3.20 \sim 3.09\left(\mathrm{~m}, 4 \mathrm{H}, \mathrm{OCH}_{2} \mathrm{CH}_{2}, \mathrm{SCH}_{2} \mathrm{CH}_{2} \mathrm{CH}_{3}\right)$, 1.74 (sextet, $J=7.2 \mathrm{~Hz}, 2 \mathrm{H}, \mathrm{SCH}_{2} \mathrm{CH}_{2} \mathrm{CH}_{3}$ ), 1.01 (t, $J=$ $\left.7.2 \mathrm{~Hz}, 3 \mathrm{H}, \quad \mathrm{SCH}_{2} \mathrm{CH}_{2} \mathrm{CH}_{3}\right) ;{ }^{13} \mathrm{C}$ NMR $(100 \mathrm{MHz}$, DMSO- $\left.d_{6}\right) \delta: 170.36,159.75,157.96,151.46,129.90$, 129.27, 123.56, 113.85, 89.64, 86.00, 72.79, 70.55, 68.57, 61.74, 54.97, 33.29, 32.65, 22.11, 13.20; IR (KBr) v: 3395, 2959, 2930, 2869, 1586, 1513, 1442, 1336, 1247, 1184, 1078, $1041 \mathrm{~cm}^{-1}$; HRMS (ESI) calcd for $\mathrm{C}_{21} \mathrm{H}_{28} \mathrm{~N}_{5} \mathrm{O}_{6} \mathrm{~S}$ $[\mathrm{M}+\mathrm{H}]^{+}$478.1760, found 478.1750 .

6-(4-氟苯乙氧基)-2-丙硫基-8-氮杂嘌呤核苷(11i): 白色固体, 产率为 $25 \%$. m.p. $93 \sim 95{ }^{\circ} \mathrm{C} ;{ }^{1} \mathrm{H}$ NMR (400 MHz, DMSO- $\left.d_{6}\right) \delta: 7.41 \sim 7.12(\mathrm{~m}, 4 \mathrm{H}, \mathrm{Ar}), 6.16(\mathrm{~d}, J=$ $\left.4.6 \mathrm{~Hz}, 1 \mathrm{H}, \mathrm{H}-1^{\prime}\right), 5.61$ (d, $\left.J=5.8 \mathrm{~Hz}, 1 \mathrm{H}, \mathrm{OH}-2^{\prime}\right), 5.31$ (d, $\left.J=5.5 \mathrm{~Hz}, 1 \mathrm{H}, \mathrm{OH}-3^{\prime}\right), 4.88 \sim 4.77$ (m, 4H, H-2', OH-5', $\mathrm{OCH}_{2} \mathrm{CH}_{2}$ ), 4.33 (q, $\left.J=4.8 \mathrm{~Hz}, 1 \mathrm{H}, \mathrm{H}-3^{\prime}\right), 4.01$ (q, $J=4.7$ $\left.\mathrm{Hz}, 1 \mathrm{H}, \mathrm{H}-4^{\prime}\right), 3.65 \sim 3.59$ (m, $\left.1 \mathrm{H}, \mathrm{H}-5^{\prime} \alpha\right), 3.51 \sim 3.45$ (m, $\left.1 \mathrm{H}, \mathrm{H}-5^{\prime} \beta\right), 3.20 \sim 3.12\left(\mathrm{~m}, 4 \mathrm{H}, \mathrm{OCH}_{2} \mathrm{CH}_{2}, \mathrm{SCH}_{2} \mathrm{CH}_{2}-\right.$ $\mathrm{CH}_{3}$ ), 1.74 (sextet, $J=7.2 \mathrm{~Hz}, 2 \mathrm{H}, \mathrm{SCH}_{2} \mathrm{CH}_{2} \mathrm{CH}_{3}$ ), 1.01 (t, $\left.J=7.3 \mathrm{~Hz}, 3 \mathrm{H}, \mathrm{SCH}_{2} \mathrm{CH}_{2} \mathrm{CH}_{3}\right) ;{ }^{13} \mathrm{C}$ NMR $(100 \mathrm{MHz}$, 
DMSO- $\left.d_{6}\right) \delta: 170.36,162.24,159.70,151.47,133.76$, 130.71, 123.53, 115.00, 89.64, 86.00, 72.79, 70.55, 68.27, 61.73, 33.28, 32.65, 22.09, 13.19; IR (KBr) v: 3363, 2962, 2928, 2869, 1586, 1510, 1442, 1336, 1221, 1186, 1077, $1050 \mathrm{~cm}^{-1}$; HRMS (ESI) calcd for $\mathrm{C}_{20} \mathrm{H}_{25} \mathrm{FN}_{5} \mathrm{O}_{5} \mathrm{~S}[\mathrm{M}+$ $\mathrm{H}]^{+}$466.1560, found 466.1551 .

6-(2-噻吩乙氧基)-2-丙硫基-8-氮杂嘌呤核苷(11j): 棕色固体, 产率为 $21 \%$. m.p. $73 \sim 75{ }^{\circ} \mathrm{C} ;{ }^{1} \mathrm{H}$ NMR (400 MHz, DMSO- $\left.d_{6}\right) \delta: 7.37$ (d, $\left.J=4.7 \mathrm{~Hz}, 1 \mathrm{H}, \mathrm{H}-5 "\right), 7.01 \sim$ 6.98 (m, 2H, H-3", H-4"), 6.16 (d, J=4.4 Hz, 1H, H-1'), $5.61\left(\mathrm{~d}, J=5.6 \mathrm{~Hz}, 1 \mathrm{H}, \mathrm{OH}-2^{\prime}\right), 5.31$ (d, $J=5.4 \mathrm{~Hz}, 1 \mathrm{H}$, $\left.\mathrm{OH}-3^{\prime}\right), 4.88 \sim 4.78\left(\mathrm{~m}, 4 \mathrm{H}, \mathrm{H}-2^{\prime}, \mathrm{OH}-5^{\prime}, \mathrm{OCH}_{2} \mathrm{CH}_{2}\right), 4.33$ (q, $\left.J=4.7 \mathrm{~Hz}, 1 \mathrm{H}, \mathrm{H}-3^{\prime}\right), 4.01$ (q, $J=4.4 \mathrm{~Hz}, 1 \mathrm{H}, \mathrm{H}-4^{\prime}$ ), $3.65 \sim 3.59\left(\mathrm{~m}, 1 \mathrm{H}, \mathrm{H}-5^{\prime} \alpha\right), 3.51 \sim 3.46\left(\mathrm{~m}, 1 \mathrm{H}, \mathrm{H}-5^{\prime} \beta\right)$, $3.41\left(\mathrm{t}, J=6.1 \mathrm{~Hz}, 2 \mathrm{H}, \mathrm{OCH}_{2} \mathrm{CH}_{2}\right), 3.24 \sim 3.13(\mathrm{~m}, 2 \mathrm{H}$, $\mathrm{SCH}_{2} \mathrm{CH}_{2} \mathrm{CH}_{3}$ ), 1.75 (sextet, $J=7.1 \mathrm{~Hz}, 2 \mathrm{H}, \mathrm{SCH}_{2} \mathrm{CH}_{2}-$ $\left.\mathrm{CH}_{3}\right), 1.01$ (t, $\left.J=7.4 \mathrm{~Hz}, 3 \mathrm{H}, \mathrm{SCH}_{2} \mathrm{CH}_{2} \mathrm{CH}_{3}\right) ;{ }^{13} \mathrm{C} \mathrm{NMR}$ $\left(100 \mathrm{MHz}, \mathrm{DMSO}-d_{6}\right) \delta: 170.36,159.64,151.51,139.44$, $127.01,125.96,124.59,123.55,89.65,86.02,72.79,70.55$, $68.05,61.73,32.67,28.48,22.12$, 13.20; IR (KBr) v: 3364, 2959, 2927, 2866, 1587, 1442, 1335, 1279, 1218, 1189, 1078, $772 \mathrm{~cm}^{-1}$; HRMS (ESI) calcd for $\mathrm{C}_{18} \mathrm{H}_{24} \mathrm{~N}_{5} \mathrm{O}_{5} \mathrm{~S}_{2}$ $[\mathrm{M}+\mathrm{H}]^{+}$454.1219, found 454.1209.

\section{3 化合物 11 的抗血小板凝聚活性测试}

抽取健康人的血液对 10 个 6 -烷氧基-2-丙硫基-8-氮 杂嘌呤核苷化合物进行了抗血小板凝聚活性测试.

血小板提取与制备：血液来自两周内未服用过抗血 小板药物的志愿者, 抽取肘部静脉血, 以 $1: 6$ (体积比) 的右旋枸椽酸 $\mathrm{ACD}(2.5 \mathrm{~g}$ 枸椽酸钠, $1.5 \mathrm{~g}$ 枸椽酸, $2 \mathrm{~g}$ 葡 萄糖溶于 $100 \mathrm{~mL}$ 去离子水中)抗凝. 混合均匀后, 常温 $300 \mathrm{~g}$ 离心 $20 \mathrm{~min}$, 分离得到富血小板血浆(platelet rich plasma, PRP). 将 PRP 在常温 $900 \mathrm{~g}$ 离心 $8 \mathrm{~min}$, 分离得 到血小板, 重悬在含 $0.01 \mu \mathrm{mol} / \mathrm{mL}$ 三磷酸腺苷双磷酸酶
(Apyrase)的苔氏液(Tyrode buffer)溶液中 (Apyrase/Tyrode buffer, $V: V=1: 2000)$. 生理盐水作为贫血小板血 浆(PPP)用以空白对照.

抗血小板聚集试验：首先将血小板聚集仪预热至 $37{ }^{\circ} \mathrm{C}$, 以 PPP 为对照, 在血小板悬液中分别加入纤维 蛋白原(Fibrinogen)以及溶于 DMSO 的待测样品(终浓度 为 $10 \mu \mathrm{mol} / \mathrm{L}), 3 \mathrm{~min}$ 后加入二磷酸腺苷 $(\mathrm{ADP})$, 测定最 大血小板凝聚率. 通过得到的血小板凝聚率评估待测化 合物对 ADP 诱导的血小板凝聚的抵抗作用. 试验过程 中, AR-C69931MX 为阳性对照物, DMSO 为阴性对照 物.

\section{2 结果与讨论}

\section{1 中间体 9-[(2', $3^{\prime}, 5^{\prime}$-三-O-苯甲酰基)- $\beta$ - $D$-呋喃核糖 基]-2-丙硫基-6-氯-8-氮杂嘌呤(10)的合成}

化合物 10 的合成中, 最初采用了 Silyl-HilbertJohnson 核苷合成法 ${ }^{[20]}$. 如 Scheme 3 所示, 通过 8-氮杂 嘌呤与 1- $O$-乙酰基-2,3,5-三- $O$-苯甲酰基- $\beta$ - $D$-呋喃核糖 在 Lewis 酸催化剂无水 $\mathrm{SnCl}_{4}$ 存在条件下反应, 得到化 合物 10. 但通过实验发现，该方法会得到嘌呤环上 7,8,9-位分别被取代的三种异构体，从而对分离提纯造 成了很大的困难，同时也降低了 9-位取代产物的产率.

为了提高化合物 10 的产率，降低其分离提纯难度， 我们尝试通过先接核糖环，然后再进行重氮化与偶联的 方法制备化合物 10. 在进行核糖环与嘧啶环的对接试 验中, 我们最开始尝试使用 Route 2 的合成方法, 通过 使用化合物 16 与化合物 17 进行亲核取代反应合成化合 物 8 (Scheme 4). 但通过实验发现, 无论是有机碱 $\mathrm{Et}_{3} \mathrm{~N}$ 或是强碱 $\mathrm{NaH}$, 反应都未能得到目标产物，其原因可能 是嘧啶及嘧啶环上取代基(硝基和卤原子)的吸电子诱导 效应及吸电子共轭效应导致氨基的亲核性降低，从而很 难发生亲核取代反应. 考虑到嘧啶环上 4-位卤素比较活 泼，容易与亲核试剂发生亲核取代反应，我们设计了
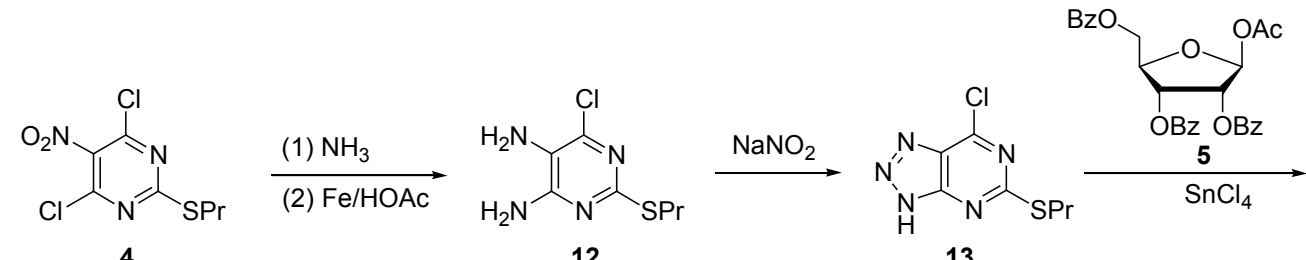

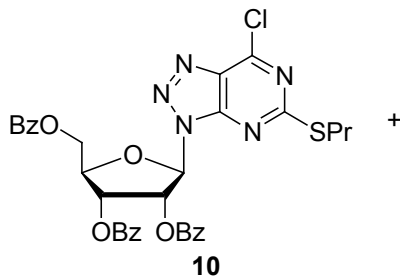

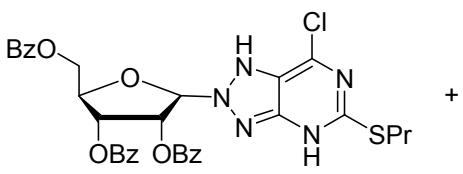

14<smiles>[R6]C1OC([R6])C(n2nnc3nc(CCCC)nc(Cl)c32)OC1COC(=O)OC</smiles>

Scheme 3 


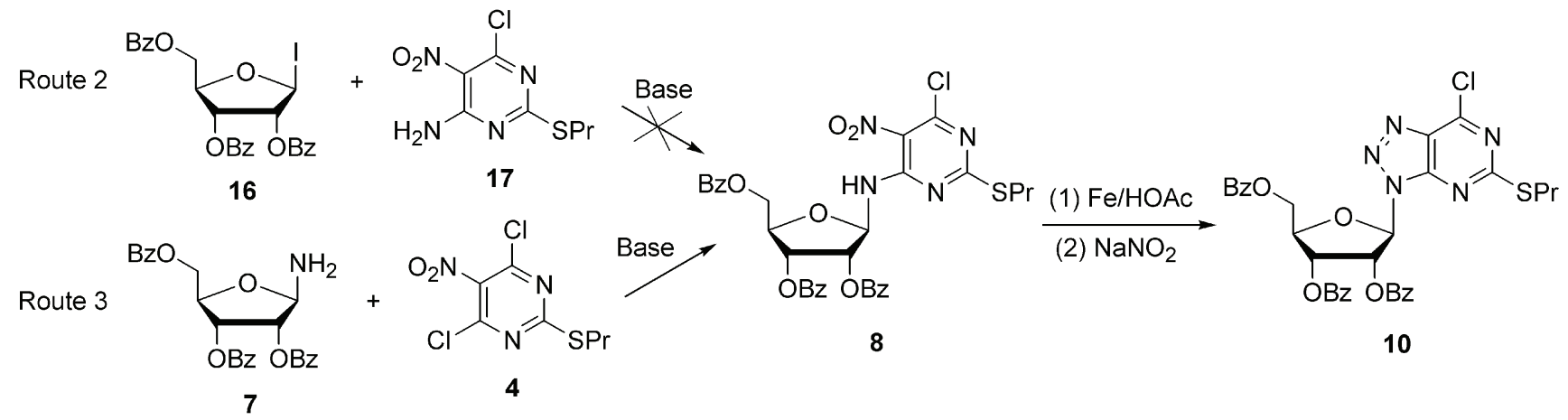

Scheme 4

Route 3 的合成路线, 在 $N, N$-二异丙基乙胺作为缚酸剂 的条件下, 化合物 7 作为亲核试剂与化合物 4 反应, 顺 利得到化合物 8 .

对比 Route 1 与 Route 3, 由于得到 3 种异构体, Route 1 制备化合物 $\mathbf{1 0}$ 的总产率仅为 $11.6 \%$, 而 Route 3 的总产率则为 $34.3 \%$. 因此我们最终选用了 Route 3 .

\section{2 化合物 $11 \mathrm{a} \sim 11 \mathrm{j}$ 的波谱分析}

以化合物 11a 为例, ${ }^{1} \mathrm{H}$ NMR 中显示在 $\delta 6.21,5.66$ 和 5.35 处存在 3 组双峰分别归属于核糖环上的 $1^{\prime}$-位氢 以及 2'-, 3'-位的羟基; $\delta 4.91,4.38$ 以及 4.05 处存在 3 组 四重峰分别归属于核糖环上 2'-, 3'-和 4'-位氢; 5'-位的羟 基则以三重峰的形式出现在 $\delta 4.83$; 由于两个氢原子的 化学不等价和受到邻位氢以及羟基氢的影响, 核糖环上 5'-位亚甲基的两个氢分别以多重峰的形式出现在 $\delta 3.53$ 和 3.66 附近; $\delta 4.20$ 处的单峰属于嘌呤环 6 位取代的 $\mathrm{OCH}_{3} ; \delta 1.05$ 的三重峰, $\delta 1.78$ 的六重峰以及 $\delta 3.21$ 附近 的多重峰则为嘌呤 2-位丙硫基的特征峰. ${ }^{13} \mathrm{C}$ NMR 中显 示, 化学位移 $\delta 32.66,22.08,13.18$ 为丙硫基的特征峰; 化学位移 $\delta 55.04$ 为嘌呤环 6 位取代的甲氧基的特征峰; 化学位移 $\delta 89.67,85.99,72.82,70.56,61.75$ 为核糖环的 特征峰; 化学位移 $\delta 170.43,160.14,151.35,123.63$ 为嘧 啶环的特征峰. 同时 HRMS (ESI) calcd for $\mathrm{C}_{13} \mathrm{H}_{20} \mathrm{~N}_{5} \mathrm{O}_{5} \mathrm{~S}$ $[\mathrm{M}+\mathrm{H}]^{+} 358.1185$, found 358.1183 表明了 11a 的结构. 同理，化合物 $11 \mathrm{~b} \sim 11 \mathrm{j}$ 的结构也经过 ${ }^{1} \mathrm{H}$ NMR, ${ }^{13} \mathrm{C}$ NMR, IR 和 HRMS 得到确认.

\section{3 抗血小板凝聚活性评价}

10 个化合物的抗血小板凝聚活性测试结果如表 1 所示. 对比 11a, 11b, 11c 和 11e 4 个化合物, 可以发现取 代基碳链越长, 化合物的抗血小板凝聚活性越好, 尤其 是当取代基为丙基时, 化合物表现出最好的抗血小板凝 聚活性. 对比 $11 \mathrm{c}, 11 \mathrm{~d}$ 与 $11 \mathrm{e}, 11 \mathrm{f}, 11 \mathrm{~g}$ 两组化合物, 当取 代基中碳的个数相同时, C-6 位被直链烷氧基取代的化 合物 11e 与 11e 比 C-6 位被支链烷氧基取代的化合物 11d, 11f, 11g 具有更好的抗血小板凝聚活性. 结果还显
示，相比于 C-6 位为烷氧基的化合物 11a 11g, C-6 位被 芳烷氧基或杂环烷氧基取代的化合物 11h 11j 表现出 更好的抗血小板凝聚活性.

表 1 化合物 $11 \mathrm{a} \sim 11 \mathrm{j}$ 的抗血小板凝聚活性

Table 1 The antiplatelet aggregation activities of compounds $11 \mathbf{a} \sim 11 \mathbf{j}$

\begin{tabular}{|c|c|c|c|}
\hline Entry & Compd. & $\mathrm{R}$ & $\mathrm{PAR}_{\max }{ }^{a} / \%$ \\
\hline 1 & 11a & $\mathrm{Me}$ & 112.50 \\
\hline 2 & 11b & Et & 93.33 \\
\hline 3 & $11 \mathrm{c}$ & $n-\operatorname{Pr}$ & 53.33 \\
\hline 4 & 11d & iso-Pr & 86.67 \\
\hline 5 & $11 \mathrm{e}$ & $n-\mathrm{Bu}$ & 67.29 \\
\hline 6 & 11f & iso-Bu & 68.57 \\
\hline 7 & $11 \mathrm{~g}$ & $s e c-\mathrm{Bu}$ & 80.00 \\
\hline 8 & $11 \mathrm{~h}$ & $p-\mathrm{MeOC}_{6} \mathrm{H}_{4} \mathrm{CH}_{2} \mathrm{CH}_{2}$ & 39.02 \\
\hline 9 & $11 \mathrm{i}$ & $p-\mathrm{FC}_{6} \mathrm{H}_{4} \mathrm{CH}_{2} \mathrm{CH}_{2}$ & 56.58 \\
\hline 10 & $11 \mathrm{j}$ & & 53.95 \\
\hline 11 & AR-C69931MX & & 0 \\
\hline
\end{tabular}

${ }^{a}$ Maximum platelet aggregation rate.

\section{References}

[1] Jennings, L. K. Am. J. Cardiol. 2009, 103, 4A.

[2] Awtry, E. H.; Loscalzo, J. Circulation 2000, 101, 1206.

[3] Cattaneo, M. Drug News Perspect 2006, 19, 253.

[4] James, E.; David, E.; Cindy, L. Circulation 2003, 108, 1316.

[5] Aerschot, A.; Mamos, P.; Weyns, J. J. Med. Chem. 1993, 36, 2938.

[6] Sagi, G.; Otvos, L.; Ikeda, S. J. Med. Chem. 1994, 37, 1307.

[7] Smith, W. C.; Sidwell, W. R.; Robins, K. R. J. Med. Chem. 1972, 15,883 .

[8] Montgomery, A. J.; Thomas, J. H. J. Med. Chem. 1972, 15, 305.

[9] Matsuda, A.; Shinozaki, M.; Yamaguchi, T. J. Med. Chem. 1992, $35,241$.

[10] Estep, G. K.; Josef, A. K.; Bacon, R. E. J. Med. Chem. 1995, 38, 2582.

[11] Cox, D.; Ingall, A.; Willis, P. WO 9703084, 1997 [Chem. Abstr. 1997, 126, 186328].

[12] Zhang, H. C.; Maryanoff, B. E.; Ye, H.; Chen, C. L. WO 2008054795, 2008 [Chem. Abstr. 2008, 148, 517743].

[13] Ding, Z. R.; Kim, S.; Kunapuli, S. P. Mol. Pharm. 2005, 69, 338.

[14] Wu, Z. J.; Li, S. L.; Ding, Z. R.; Du, H. G. Chin. J. Org. Chem. 2011, 31, 1618 (in Chinese). 
(吴兆军, 李顺来, 丁忠仁, 杜洪光, 有机化学, 2011, 31, 1618.)

[15] He, Q. W.; Du, H. G. Chin. J. Org. Chem. 2012, 32, 1678 (in Chinese).

(何琦文, 杜洪光, 有机化学, 2012, 32, 1678.)

[16] Du, H. G.; Chen, L.; Wang, S. M. CN 102250097, 2011 [Chem. Abstr. 2011, 155, 683474].

[17] Bonnert, R.; Ingall, A.; Springthorpe, B.; Willis, P. WO 9828300 ,
1998 [Chem. Abstr. 1998, 129, 95506].

[18] Storer, R.; Gosselin, G.; Griffon, J. F.; Pierra, C. WO 2007144686 , 2006 [Chem. Abstr. 2006, 148, 79268].

[19] Volpini, R.; Marucci, G.; Buccioni, M.; Ben, D. D.; Lambertucci, C.; Lammi, C.; Mishra, R. C.; Thomas, A.; Cristalli, G. ChemMedChem 2011, 6, 1074.

[20] Niedballa, U.; Vorbrüggen, H. J. Org. Chem. 1976, 41, 2084.

(Li, L.; Fan, Y.) 